\title{
ON DYNAMICS OF STRINGS AND BRANES
}

\author{
A.V. Golovnev, L.V. Prokhorov \\ V.A. Fock Institute of Physics \\ Sankt-Petersburg State University
}

\begin{abstract}
We study Nambu-Goto strings and branes. It is shown that they can be considered as continuous limits of ordered discrete sets of relativistic particles for which the tangential velocities are excluded from the action. The linear in unphysical momenta constraints are found. It allows to derive the evolution operators for the objects under consideration from the "first principles".
\end{abstract}

\section{Introduction.}

We consider Nambu-Goto strings and branes. We start with the simplest case of one relativistic particle which formally can be considered as a 0-brane. The relativistic invariant form of its action is raparametrization invariant and has a constraint, commonly taken in a quadratic form with the loss of information on the momentum sign. It led to some problems [1,2] in quantum description of free relativistic particles. In section 2 we explain how these problems can be overcome [3,4] using a linear "constraint" containing the sign of the velocity $\frac{\partial x^{0}}{\partial \sigma_{0}}$ ( $\frac{\partial t}{\partial \tau}$ in usual notations) and fixing the physical sector of quantum theory by the condition $\frac{\partial x^{0}}{\partial \sigma_{0}}>0$. After that the operator of evolution (and propagator [3]) can be derived from the "first principles" of quantum mechanics.

In sections 3 and 4 we prove that p-branes may be considered as continuous limits of discrete sets of relativistic particles and that the $p$-brane action

$$
S=-\gamma \int d^{p+1} \sigma \sqrt{(-1)^{p} g}, \quad g=\operatorname{det} \frac{\partial x^{\mu}}{\partial \sigma_{a}} \frac{\partial x_{\mu}}{\partial \sigma_{b}},
$$

is a continuous limit of sum of properly modified relativistic particle actions. Here $a, b=0,1, \ldots, p$ and $\mu=0,1, \ldots, n$ where $p+1$ and $n+1$ are the brane worldsheet and the bulk space dimentions respectively; $g$ is the induced metric determinant on the worldsheet. Strings correspond to the $p=1$ case in (11). The modification is such that particle motions along the brane hypersurface become unphysical. It yields $p$ constraints; the remaining constraint $(H=0)$ is a consequence of arbitrariness of "time" $\sigma_{0}$. 
In sections 5 and 6 we consider dynamics of strings $(p=1)$ and branes $(p>1)$. The theories are reparametrization invariant for all $p, n>p$; each of them has $p+1$ constraints. Usualy one of these constraints physicists take in a quadratic form. It causes the same problems as in the case of a single particle. We found that the operator of evolution can be constructed in the same way as in section 2 ; new difficulties are rather technical than conceptual ones. The solution is also similar to the $p=0$ case (a particle).

\section{Relativistic particle.}

Motion of free relativistic particle is defined by the well-known action

$$
S=-m \int \sqrt{1-\vec{v}^{2}} d t
$$

where $\vec{v}=\frac{d \vec{x}(t)}{d t}$. The canonical momentum is $\overrightarrow{\mathfrak{p}} \equiv \frac{\partial L}{\partial \vec{v}}=\frac{m \vec{v}}{\sqrt{1-\vec{v}^{2}}} \equiv E_{\mathfrak{p}} \vec{v}$ with $L$ being the Lagrangian, and the Hamiltonian is $H=E_{\mathfrak{p}}=\sqrt{m^{2}+\overrightarrow{\mathfrak{p}}^{2}}$. We can write down the action in the explicitly relativistic invariant form by parametrization of the world line: $x^{\mu}=x^{\mu}\left(\sigma_{0}\right)$ (usually one uses $\tau$ instead of $\left.\sigma_{0}\right)$ with $x^{\mu}\left(\sigma_{0}\right)=\left(t\left(\sigma_{0}\right), \vec{x}\left(\sigma_{0}\right)\right), \mu=0, \ldots, n$. We denote $\dot{x}^{\mu} \equiv \frac{d x^{\mu}}{d \sigma_{0}}$, so that $\vec{v}=\dot{\vec{x}} \frac{d \sigma_{0}}{d t}$ and

$$
S=-m \int \sqrt{\dot{x}^{\mu} \dot{x}_{\mu}} d \sigma_{0}
$$

It is the $p=0$ case of (11) in which we put $m$ (particle's mass) instead of $\gamma$. The vector of canonical momentum is

$$
\mathfrak{p}_{\mu} \equiv \frac{\partial L}{\partial \dot{x}^{\mu}}=-m \frac{\dot{x}_{\mu}}{\sqrt{\dot{x}^{2}}} .
$$

The obtained theory is invariant under reparametrization group $\sigma_{0} \rightarrow$ $\tilde{\sigma}_{0}=f\left(\sigma_{0}\right)$ ), hence its Hamiltonian is zero and by squaring the equation (2) one gets a constraint:

$$
\mathfrak{p}^{2}-m^{2}=0 .
$$

Of course, the information about the sign of $\mathfrak{p}_{0}$ is lost. On the other hand one can prove that any constraint has to be linear in unphysical momenta $[3,4]$. It was a serious obstruction to the $a b$ initio derivation of relativistic particle 
propagator (authors of $[1,2]$ used some additional assumptions). The problem was overcome only in [3]. One can obtain the solution in the following way.

From the constraint (3) one finds $\mathfrak{p}_{0}= \pm \sqrt{m^{2}+\overrightarrow{\mathfrak{p}}^{2}}$, and it is obvious from (2) that sign $\mathfrak{p}_{0}=-\operatorname{sign} \dot{x}_{0}$. Combining these facts we have

$$
\mathfrak{p}_{0}+E_{\mathfrak{p}} \operatorname{sign}\left(\dot{x}^{0}\right)=0
$$

with $E_{\mathfrak{p}}(\overrightarrow{\mathfrak{p}})=\sqrt{m^{2}+\overrightarrow{\mathfrak{p}}^{2}}$. The Hamiltonian is zero and the total Hamiltonian $[5]$ is

$$
H_{T}=v\left(\mathfrak{p}_{0}+E_{\mathfrak{p}} \operatorname{sign}\left(\dot{x}^{0}\right)\right) .
$$

Here $v$ is the Lagrange multiplier. Strictly speaking Eq. (4) is not a constraint because it contains velocity (and $H_{T}$ is not a Hamiltonian due to the same reason). But it depends only upon the sign of $\dot{x}$, and this fact allows us to formulate the quantum theory.

We fix the $\sigma_{0}$ "time arrow" by condition

$$
\frac{\partial x^{0}}{\partial \sigma_{0}}>0
$$

which forces us to admit that $v>0$ (because $\Delta x^{0}=v \Delta \sigma_{0}$, see later). We use the evolution operator

$$
\begin{gathered}
U_{\omega}(x, \tilde{x})=\left\langle x\left|\exp \left(-i \omega \hat{H}_{T}\right)\right| \tilde{x}\right\rangle, \\
\psi\left(x, \sigma_{0}+\omega\right)=\int d^{4} \tilde{x} U_{\omega}(x, \tilde{x}) \psi\left(\tilde{x}, \sigma_{0}\right)
\end{gathered}
$$

and the following relation for infinitesimal $\omega$ :

$$
\left\langle x\left|\exp \left(-i \omega \hat{H}_{T}\right)\right| \tilde{x}\right\rangle=\int d^{4} \mathfrak{p}\left\langle x\left|\exp \left(-i \omega H_{T}(x, \mathfrak{p})\right)\right| \mathfrak{p}\right\rangle\langle\mathfrak{p} \mid \tilde{x}\rangle .
$$

Integrating over $\mathfrak{p}_{0}$ and (with use of $\delta$-function $\delta\left(x^{0}-\tilde{x}^{0}-\omega v\right)$ ) over $\tilde{x}_{0}$ we get for the wave function [4]:

$$
\psi\left(x^{0}, \vec{x}\right)=\int \frac{d^{3} \mathfrak{p} d^{3} \tilde{x}}{(2 \pi)^{3}} \exp \left(i\left[\mathfrak{p}_{i} \Delta x^{i}-\Delta x^{0} E_{\mathfrak{p}}\right]\right) \psi\left(\tilde{x}^{0}, \overrightarrow{\tilde{x}}\right),
$$

where $\Delta x^{0}=v \omega$, and we omit the argument $\sigma_{0}$ because all the information on $\sigma_{0}$ is accumulated in $x^{0}$. Using (6) one can get the right Feynman propagator for a relativistic particle [4]. 


\section{String as an ordered system of relativistic particles.}

Now we show that any string and brane can be described as a system of particles. More precisely, the action (11) may be regarded as a continuous limit of sum of free relativistic particle actions provided that we take $\vec{v}_{\perp}$ instead of $\vec{v}$, where $\vec{v}_{\perp}$ is the part of velocity orthogonal to the constant time hypersurface of the brane worldsheet. We deal here with a kind of indirectly introduced particle interaction.

In this section we consider a string (1-brane) and reproduce the proof of our statement by an explicit calculation [6]. We consider $N+1$ particles with the position vectors $\vec{x}_{k}\left(x^{0}\right), k=0,1, \ldots, N$ and the action

$$
S=-m \sum_{k=0}^{N} \int d x^{0} \sqrt{1-\vec{v}_{k \perp}^{2}\left(x^{0}\right)}
$$

in which $\vec{v}_{k}=\frac{d \vec{x}_{k}}{d x^{0}}$ and

$$
\vec{v}_{k \perp}=\vec{v}_{k}-\frac{\left(\vec{v}_{k} \cdot \Delta \vec{x}_{k}\right)}{\left(\Delta \vec{x}_{k}\right)^{2}} \Delta \vec{x}_{k}, \Delta \vec{x}_{k} \equiv \vec{x}_{k+1}-\vec{x}_{k} .
$$

In the continuous limit we define $\frac{k A}{N} \rightarrow \sigma_{1}, \frac{\Delta \vec{x}_{k}}{A / N} \rightarrow \vec{k}\left(\sigma_{1}\right), \frac{m}{\left|\Delta \vec{x}_{k}\right|} \rightarrow \gamma$. Here $\sigma_{1} \in[0, A]$; usually one takes $A=\pi$, but for our purposes it may be natural to consider $A$ as the string length and $\left|\Delta \vec{x}_{k}\right|=\frac{A}{N},|\vec{k}|=1$. In any case we have

$$
S=-\gamma \sum_{k=0}^{N} \int d x^{0} \Delta l \sqrt{1-\vec{v}_{k \perp}^{2}} \rightarrow-\gamma \int d x^{0}|\vec{k}| d \sigma_{1} \sqrt{1-\vec{v}_{\perp}^{2}}
$$

with $\vec{k}=\frac{\partial \vec{x}\left(x^{0}, \sigma_{1}\right)}{\partial \sigma_{1}}, \quad \vec{v}=\frac{\partial \vec{x}\left(x^{0}, \sigma_{1}\right)}{\partial x^{0}}$ and $\vec{v}_{\perp}=\vec{v}-\frac{(\vec{v} \vec{k})}{k^{2}} \vec{k}$. The string length is $L=\int|\vec{k}| d \sigma_{1}$. After that we parametrize the worldsheet $x^{0}=$ $x^{0}\left(\sigma_{0}, \sigma_{1}\right), \vec{x}=\vec{x}\left(\sigma_{0}, \sigma_{1}\right)$ introducing a new parameter $\sigma_{0}$ (again the standard notations are $x^{0} \rightsquigarrow t$ and $\left.\sigma_{0} \rightsquigarrow \tau\right)$. We have $\dot{x} \equiv \frac{\partial x\left(\sigma_{0}, \sigma_{1}\right)}{\partial \sigma_{0}}=(1, \vec{v}) \dot{x}_{0}$, $x^{\prime} \equiv \frac{\partial x\left(\sigma_{0}, \sigma_{1}\right)}{\partial \sigma_{1}}=\left(x_{0}{ }^{\prime}, \vec{k}+\vec{v} x_{0}{ }^{\prime}\right)$ and

$$
S=-\gamma \int d^{2} \sigma \dot{x}^{0}|\vec{k}| \sqrt{1-\vec{v}_{\perp}^{2}}
$$


The last expression is equal to the Nambu-Goto action:

$$
S=-\gamma \int d^{2} \sigma \sqrt{\left(\dot{x} x^{\prime}\right)^{2}-\dot{x}^{2} x^{\prime 2}}
$$

Of course, one could start with it and get $S=-\gamma \int d x^{0} d l \sqrt{1-\vec{v}_{\perp}^{2}}$ which is a continuous limit of (17).

We can also propose another discrete analogue of the Nambu-Goto string. Let's consider the following action:

$$
S=-m \sum_{k=0}^{N} \int d \sigma_{0} \sqrt{\dot{x}_{k \perp}^{\mu} \dot{x_{\mu_{k \perp}}}}
$$

where $\dot{x}_{k \perp}^{\mu}$ is the part of $\dot{x}_{k}^{\mu}$ perpendicular to $x_{k+1}^{\mu}-x_{k}^{\mu}$. The continuous limit is $N \rightarrow \infty, \frac{k A}{N} \rightarrow \sigma_{1}, \frac{m}{\left|\Delta s_{k}\right|} \rightarrow \gamma$ with the invariant interval $\left(\Delta s_{k}\right)^{2}=$ $\left(x_{k+1}^{\mu}-x_{k}^{\mu}\right)\left(x_{\mu_{k+1}}-x_{\mu_{k}}\right)$. We have $\dot{x}_{\perp}^{\mu}=\dot{x}^{\mu}-\frac{\dot{x}^{\nu} x_{\nu}^{\prime}}{x^{\prime} \rho_{\rho}^{\prime}} x^{\prime \mu},\left(\frac{d s}{d \sigma_{1}}\right)^{2}=x^{\prime 2}$, and

$$
S=-\gamma \int d \tau|d s| \sqrt{\dot{x}^{2}-\frac{\left(\dot{x} x^{\prime}\right)^{2}}{x^{\prime 2}}}=-\gamma \int d \sigma_{0} d \sigma_{1} \sqrt{\left(\dot{x} x^{\prime}\right)^{2}-\dot{x}^{2} x^{\prime 2}} .
$$

In contrast to the previous paragraph the presented discrete theory has the relativistic invariant form from the very begining but even the sense of $\dot{x}_{\perp}$ depends upon the parametrization of the worldsheet. In the gauge $\sigma_{0}=x^{0}$ these two approaches coincide.

\section{Brane as an ordered system of relativistic particles.}

Now we shall prove our statement for $p>1$. We consider $(N+1)^{p}$ particles arranged into some $p$-dimensional lattice with the position vectors $\vec{x}_{i_{1} i_{2} \ldots i_{p}}$ and the action

$$
S=-m \int d x^{0} \sum_{i_{1}=0}^{N} \cdots \sum_{i_{p}=0}^{N} \sqrt{1-\vec{v}_{i_{1}, \ldots i_{p} \perp}^{2}}
$$

where $\vec{v}_{i_{1}, \ldots i_{p} \perp}$ is the component of $\vec{v}_{i_{1} \ldots i_{p}}$ perpendicular to $\vec{x}_{i_{1} \ldots i_{k}+1 \ldots i_{p}}-$ $\vec{x}_{i_{1} \ldots i_{k} \ldots i_{p}}$ for all $k$. In continuous limit we demand $\frac{A i_{k}}{N} \rightarrow \sigma_{k}$ and $\frac{m}{\Delta V} \rightarrow \gamma$ 
with $\Delta V$ being volume of a cell of the lattice. The action takes the form $S=-\gamma \int d x_{0} d V \sqrt{1-\vec{v}_{\perp}^{2}}$, and we need to prove that $S$ is equal to (11).

For the sake of simplicity first we consider some special coordinate system on the brane. Let the brane be parametrized by $\sigma_{0}, \sigma_{1}, \ldots, \sigma_{p}, \quad \sigma_{i} \in[0, A]$ for $i=1, \ldots, p$. We choose a coordinate system in which $\sigma_{0}=x^{0}$, so that $\frac{\partial x^{0}}{\partial \sigma_{i}}=0$, and $\frac{\partial x^{\mu}}{\partial \sigma_{i}} \frac{\partial x_{\mu}}{\partial \sigma_{j}}=0, i \neq j$. It is always possible, locally at least. Let's denote $\vec{k}_{i} \equiv \frac{\partial \vec{x}\left(x^{0}, \sigma_{i}\right)}{\partial \sigma_{i}}$ and $\vec{v} \equiv \frac{\partial \vec{x}\left(x^{0}, \sigma_{i}\right)}{\partial x^{0}}$ on the worldsheet. In our coordinate system $\vec{k}_{i}$ is orthogonal to $\vec{k}_{j}, i \neq j$ and

$$
\vec{v}_{\perp}=\vec{v}-\sum_{i=1}^{p} \frac{\left(\vec{v} \vec{k}_{i}\right)}{k_{i}^{2}} \vec{k}_{i}
$$

Of course, one can find $\vec{v}_{\perp}$ without the orthogonality condition with the use of standard orthogonalization procedure, but in section 6 more simple proof is presented.

We have $\frac{\partial x^{\mu}}{\partial \sigma_{0}}=(1, \vec{v})$ and $\frac{\partial x^{\mu}}{\partial \sigma_{i}}=\left(0, \vec{k}_{i}\right)$, so the determinant in (11) is

$$
\begin{aligned}
\operatorname{det} \frac{\partial x^{\mu}}{\partial \sigma_{a}} \frac{\partial x_{\mu}}{\partial \sigma_{b}}=\left|\begin{array}{ccccc}
1-\vec{v}^{2} & -\vec{v} \vec{k}_{1} & -\vec{v} \vec{k}_{2} & \ldots & -\vec{v} \vec{k}_{p} \\
-\vec{v} \vec{k}_{1} & -k_{1}^{2} & 0 & \ldots & 0 \\
-\vec{v} \vec{k}_{2} & 0 & -k_{2}^{2} & \ldots & 0 \\
\vdots & \vdots & \vdots & \ddots & \vdots \\
-\vec{v} \vec{k}_{p} & 0 & 0 & \ldots & -k_{p}^{2}
\end{array}\right|= \\
\quad=\left(\prod_{i=1}^{p}\left(-k_{i}{ }^{2}\right)\right)\left(1-\vec{v}^{2}+\sum_{i=1}^{p} \frac{\left(\vec{v} \vec{k}_{i}\right)^{2}}{k_{i}{ }^{2}}\right)
\end{aligned}
$$

where $a, b=0,1, \ldots, p$. It is not also difficult to see that the volume element at the constant time hypersurface on the brane worldsheet is $d V=\prod_{i=1}^{p}\left|k_{i}\right| d \sigma_{i}$. We conclude that the action

$$
S=-\gamma \int d \sigma_{0} d^{n} \sigma_{i} \sqrt{\left|\operatorname{det} \frac{\partial x^{\mu}}{\partial \sigma_{a}} \frac{\partial x_{\mu}}{\partial \sigma_{b}}\right|}=-\gamma \int d x_{0} d V \sqrt{1-\vec{v}_{\perp}^{2}} .
$$

It proves our statement. 


\section{Dynamics of strings.}

As it was mentioned in the Introduction, the evolution operator $U_{\omega}$ can be constructed for strings and branes in the same way as in section 2 [7]. For $p=1$ action (11) is the action of free bosonic string with the Lagrange density $[6,8-11]$

$$
\mathcal{L}=-\gamma \sqrt{-g}=-\gamma \sqrt{\left(\dot{x} x^{\prime}\right)^{2}-\dot{x}^{2} x^{\prime 2}}
$$

We assume that $\dot{x}$ is timelike and $x^{\prime}$ is spacelike, so that $\sigma_{0}$ can be regarded as a time parameter. In this case the momentum

$$
\mathfrak{p}_{\mu} \equiv \frac{\partial \mathcal{L}}{\partial \dot{x}^{\mu}}=-\gamma \frac{\left(\dot{x} x^{\prime}\right) x_{\mu}^{\prime}-x^{\prime 2} \dot{x}_{\mu}}{\sqrt{\left(\dot{x} x^{\prime}\right)^{2}-\dot{x}^{2} x^{\prime 2}}}
$$

will also be timelike. One easily gets two constraints

$$
\begin{aligned}
\mathfrak{p}_{\mu} x^{\prime \mu} & =0, \\
\mathfrak{p}^{2}+\gamma^{2} x^{\prime 2} & =0 .
\end{aligned}
$$

The second one is obtained by squaring the Eq. (10) and hence some information is lost. As in the case of a pointlike particle Eq. (12) yields $\mathfrak{p}_{0}= \pm E_{\mathfrak{p}}$ with $E_{\mathfrak{p}}=\sqrt{\overrightarrow{\mathfrak{p}}^{2}-\gamma^{2} x^{\prime 2}}$. The sign of $\mathfrak{p}_{0}$ follows from the definition of the momentum. We have

$$
\mathfrak{p}_{0}+E_{\mathfrak{p}}(x, \overrightarrow{\mathfrak{p}}) \operatorname{sign}\left(y_{0}\right)=0
$$

where $y_{\mu}=\left(\dot{x} x^{\prime}\right) x_{\mu}^{\prime}-x^{\prime 2} \dot{x}_{\mu}$. The vector $y$ is obviously timelike (indeed, $y^{2}=x^{\prime 2} g>0$ and $y_{\mu} \dot{x}^{\mu}=-g>0$, so that $\left.\operatorname{sign} \dot{x}^{0}=\operatorname{sign} y^{0}\right)$. We get the "constraint" analogous to (4):

$$
\mathfrak{p}_{0}+E_{\mathfrak{p}}(x, \overrightarrow{\mathfrak{p}}) \operatorname{sign}\left(\dot{x}^{0}\right)=0
$$

The "Hamiltonian" of the theory $H=\mathfrak{p}_{0}+E_{\mathfrak{p}} \operatorname{sign} \dot{x}^{0}$ is zero, and demanding again sign $\dot{x}^{0}>0$ we get the total Hamiltonian $H_{T}=u\left(\mathfrak{p}_{0}+E_{\mathfrak{p}}\right)+v \mathfrak{p}_{\mu} x^{\prime \mu}$. We have two unphysical momenta and need to exclude them from $E_{\mathfrak{p}}$. Let's exclude $\mathfrak{p}_{0}$ and $\mathfrak{p}_{1}$ (we denote the remaining components by the lower index 
$">$ ": $\left.\mathfrak{p}^{\mu}=\left(\mathfrak{p}_{0}, \mathfrak{p}_{1}, \mathfrak{p}_{>}\right)\right)$. One can find $\mathfrak{p}_{1}$ from (11) if $x_{1}^{\prime} \neq 0$. In [7] we restricted ourselves to the case $x_{0}^{\prime}=0$ and had

$$
E_{\mathfrak{p}}\left(x, \mathfrak{p}_{>}\right)=\sqrt{\left(\frac{\mathfrak{p}_{>} x_{>}^{\prime}}{x_{1}^{\prime}}\right)^{2}+\mathfrak{p}_{>}^{2}-\gamma^{2} x^{\prime 2}}
$$

In general case one can substitute $\mathfrak{p}_{1}$ from (11) to (12) and get a quadratic equation for $\mathfrak{p}_{0}$ :

$$
\mathfrak{p}_{0}^{2}\left(1-\left(\frac{x^{\prime 0}}{x^{\prime 1}}\right)^{2}\right)+2 \mathfrak{p}_{0} \frac{\left(\mathfrak{p}_{>} x_{>}^{\prime}\right) x^{\prime 0}}{\left(x^{\prime 1}\right)^{2}}-\mathfrak{p}_{>}^{2}\left(1+\left(\frac{x_{>}^{\prime}}{x^{\prime 1}}\right)^{2}\right)+\gamma^{2} x^{\prime 2}=0 .
$$

If $\left|x^{\prime 1}\right|>\left|x^{\prime 0}\right|$, it has two real roots of opposite signs, and we can choose a proper one following (13). Otherwise we have to try to exclude another component of $\mathfrak{p}_{\mu}$ from $E_{\mathfrak{p}}$. It's always possible because $x^{\prime}$ is spacelike and $\left|\overrightarrow{x^{\prime}}\right|>\left|x^{\prime 0}\right|$. In section 3 we have seen that the unphysical degree of freedom is related to the motion of particles along the string.

Now we write down the evolution equation (see section 2)

$$
\begin{aligned}
& \psi(x)=\int D^{n+1} \tilde{x}\left(\sigma_{1}\right)\left\langle x\left(\sigma_{1}\right)\left|\exp \left(-i \omega \hat{H}_{T}\right)\right| \tilde{x}\left(\sigma_{1}\right)\right\rangle \psi\left(\tilde{x}\left(\sigma_{1}\right)\right)= \\
& =\int D^{n+1} \mathfrak{p} D^{n+1} \tilde{x} \exp \left(i\left[\mathfrak{p}_{\mu} \Delta x^{\mu}-\omega u\left(\mathfrak{p}_{0}+E_{\mathfrak{p}}\left(\tilde{x}, \mathfrak{p}_{\perp}\right)\right)-\omega v \mathfrak{p}_{\mu} x^{\prime \mu}\right]\right) \psi(\tilde{x})= \\
& =\int D^{n-1} \mathfrak{p}_{>} D^{n+1} \tilde{x} \exp \left(i\left[-\mathfrak{p}_{>} \Delta x_{>}-\omega u E_{\mathfrak{p}}\left(\tilde{x}, \mathfrak{p}_{>}\right)+\omega v \mathfrak{p}_{>} x_{>}^{\prime}\right]\right) \times \\
& \times \delta\left(\Delta x^{0}-\omega u-\omega v x^{\prime 0}\right) \delta\left(-\Delta x_{1}+\omega v x_{1}^{\prime}\right) \psi(\tilde{x}) .
\end{aligned}
$$

Here $D \tilde{x}$ and $D \mathfrak{p}$ denote differentials in the functional spaces and all the integrals are functional ones (path integrals).

$\delta$-Functions determine the Lagrange multipliers $\omega v=\frac{\Delta x_{1}}{x_{1}^{\prime}}$ and $\omega u=$ $\Delta x^{0}-\frac{x^{\prime 0} \Delta x_{1}}{x_{1}^{\prime}}$ yielding the final result

$$
\begin{aligned}
\psi(x)=\int D^{n-1} \mathfrak{p}_{>} D^{n-1} \tilde{x}_{>} & \exp \left(i \left[-\mathfrak{p}_{>} \Delta x_{>}-\right.\right. \\
& \left.\left.-\left(\Delta x^{0}-\frac{x^{\prime 0} \Delta x_{1}}{x_{1}^{\prime}}\right) E_{p}\left(\tilde{x}, \mathfrak{p}_{>}\right)+\frac{\Delta x_{1}}{x_{1}^{\prime}} \mathfrak{p}_{>} x_{>}^{\prime}\right]\right) \psi(\tilde{x}) .
\end{aligned}
$$

If $x^{\prime 0}=0$, Eq. (15) implies $u>0$. 


\section{Dynamics of branes.}

We turn to the general case of action (11). We denote the $\sigma_{0}, \sigma_{1}, \ldots, \sigma_{p}$ derivatives of $x$ by $x_{, 0}, x_{, 1}, \ldots, x_{, p}$. Again we assume that the vector $x_{, 0}^{\mu}$ is timelike, and vectors $x_{, i}^{\mu}$ are spacelike (here and hereafter in this chapter $i, k, l=1, \ldots, p$ while $a, b=0, \ldots, p)$. Now in the action (11)

$$
g(\sigma)=\frac{1}{(p+1) !} \epsilon_{a_{0} \ldots a_{p}} \epsilon^{b_{0} \ldots b_{p}} x_{\alpha_{0}, b_{0}} x^{\alpha_{0}, a_{0}} \cdots x_{\alpha_{p}, b_{p}} x^{\alpha_{p}, a_{p}}
$$

with $\epsilon$ being the unit antisymmetric Levi-Civita symbol, and the canonical momentum is

$$
\mathfrak{p}_{\mu}=\frac{-\gamma(-1)^{p}}{p ! \sqrt{(-1)^{p} g}} \epsilon_{0 a_{1} \ldots a_{p}} \epsilon^{b_{0} \ldots b_{p}} x_{\mu, b_{0}} x_{, b_{1}} \cdot x^{, a_{1}} \ldots x_{, b_{p}} \cdot x^{, a_{p}} .
$$

Evidently $\mathfrak{p}_{\mu} x_{, i}^{\mu}=0$ due to antisymmetry of $\epsilon$, and using the equality

$$
\epsilon^{a_{0} \ldots a_{p}} g(\sigma)=\epsilon^{b_{0} \ldots b_{p}} x_{, b_{0}} \cdot x^{, a_{0}} \ldots x_{, b_{p}} \cdot x^{a_{p}}
$$

one obtains $\mathfrak{p}^{2}=(-1)^{p} \gamma^{2} \zeta(x), \quad \zeta(x)=\operatorname{det} x_{, i}^{\mu} x_{\mu, k}$. So, with the loss of information about the sign of $\mathfrak{p}_{0}$, the constraints are

$$
\begin{array}{r}
\mathfrak{p}_{\mu} x_{, i}^{\mu}=0, \quad i=1,2, \ldots, p \\
\mathfrak{p}^{2}-(-1)^{p} \gamma^{2} \zeta(x)=0 .
\end{array}
$$

From (15) we have $\mathfrak{p}_{0}= \pm E_{\mathfrak{p}}$ with $E_{\mathfrak{p}}=\sqrt{\overrightarrow{\mathfrak{p}}^{2}+(-1)^{p} \gamma^{2} \zeta(x)}$. Again, the $\mathfrak{p}_{0}$ sign can be easily found: $\mathfrak{p}_{\mu}$ and $\dot{x}_{\mu}$ are timelike and $\mathfrak{p}_{\mu} \dot{x}^{\mu}=-\gamma \sqrt{(-1)^{p} g}<0$, hence $\operatorname{sign}\left(\mathfrak{p}_{0}\right)=-\operatorname{sign}\left(\dot{x}_{0}\right)$. The result is similar to (4):

$$
\mathfrak{p}_{0}+E_{\mathfrak{p}} \operatorname{sign}\left(\dot{x}^{0}\right)=0
$$

the "Hamiltonian" is equal to zero and the total Hamiltonian $H_{T}=u\left(\mathfrak{p}_{0}+\right.$ $\left.E_{\mathfrak{p}}(x, \overrightarrow{\mathfrak{p}})\right)+v_{i} \mathfrak{p}_{\mu} x_{, i}^{\mu}$

Here $\mathrm{p}+1$ momenta are unphysical ones. We assume that $\operatorname{det}\left(x_{i, k}\right) \neq 0$ $\left(x_{i, k}\right.$ is an $p \times p$ matrix) and exclude momenta $\mathfrak{p}_{1}, \ldots, \mathfrak{p}_{p}$ from $E_{\mathfrak{p}}$. Due to (14) we have $\mathfrak{p}_{i}=\left(\left[x_{.,}\right]^{-1}\right)_{i l}\left(\mathfrak{p}_{0} x_{0, l}-\mathfrak{p}_{>} x_{>, l}\right)$. Here we denoted all the components of $\mathfrak{p}^{\mu}$ with $\mu>p$ by the lower index " >", and $\left(\left[x_{.,}\right]^{-1}\right)_{i l} \equiv d_{i l}$ is a matrix inverse of $x_{l, i}$. Then (15) turns into quadratic equation

$$
\begin{aligned}
\mathfrak{p}_{0}^{2}\left(1-d_{i l} x_{0, l} d_{i k} x_{0, k}\right)+2 \mathfrak{p}_{0} d_{i l}\left(\mathfrak{p}_{>} x_{>, l}\right) d_{i k} x_{0, k}- \\
-d_{i l}\left(\mathfrak{p}_{>} x_{>, l}\right) d_{i k}\left(\mathfrak{p}_{>} x_{>, k}\right)-(-1)^{p} \gamma^{2} \zeta(x)=0 .
\end{aligned}
$$


It has two real roots of opposite signs if and only if

$$
d_{i l} x_{0, l} d_{i k} x_{0, k}<1 .
$$

The sufficient condition is that the norm of $x_{i, k}$ as a linear operator is greater than the length of $p$-dimensional vector $x_{0, l}$. If $x_{0, l}=0$ the simple answer exists:

$$
E_{\mathfrak{p}}=E_{\mathfrak{p}}\left(x, \mathfrak{p}_{>}\right)=\sqrt{\sum_{i=1}^{p}\left(\mathfrak{p}_{i}\left(x, \mathfrak{p}_{>}\right)\right)^{2}+\mathfrak{p}_{>}^{2}+(-1)^{p} \gamma^{2} \zeta(x)} .
$$

For the wave function we have path integrals (taking (5D) into account)

$$
\begin{aligned}
& \psi(x)=\int D^{n+1} \mathfrak{p} D^{n+1} \tilde{x} \exp \left(i \left[\mathfrak{p}_{\mu} \Delta x^{\mu}-\right.\right. \\
&\left.\left.-\omega u\left(\mathfrak{p}_{0}+E_{\mathfrak{p}}\left(\tilde{x}, \mathfrak{p}_{\perp}\right)\right)-\omega v_{i} \mathfrak{p}_{\mu} x_{, i}^{\mu}\right]\right) \psi(\tilde{x})= \\
&=\int D^{n-p_{\mathfrak{p}}} D^{n+1} \tilde{x} \exp \left(i\left[-\mathfrak{p}_{>} \Delta x_{>}-\omega u E_{\mathfrak{p}}\left(\tilde{x}, \mathfrak{p}_{>}\right)+\omega v_{i} \mathfrak{p}_{>} x_{>, i}\right]\right) \times \\
& \quad \times \delta\left(\Delta x^{0}-\omega u-\omega v_{i} x_{0, i}^{\prime}\right) \prod_{l=1}^{r} \delta\left(-\Delta x_{l}+\omega v_{i} x_{l, i}\right) \psi(\tilde{x}) .
\end{aligned}
$$

Again $\delta$-functions determine the Lagrange multipliers

$$
\begin{array}{r}
\omega v_{i}=d_{i l} \Delta x_{l}, \\
\omega u=\Delta x^{0}-\omega v_{i} x_{0, i}
\end{array}
$$

and reduce the number of integrals over $x$ :

$$
\begin{aligned}
\psi(x)=\int D^{n-p} \mathfrak{p}_{>} & D^{n-p} \tilde{x}_{>} \exp \left(i \left[-\mathfrak{p}_{>} \Delta x_{>}-\right.\right. \\
& \left.\left.-\left(\Delta x^{0}-d_{i l}\left(\Delta x_{l}\right) x_{0, i}\right) E_{\mathfrak{p}}\left(\tilde{x}, \mathfrak{p}_{>}\right)+d_{i l}\left(\Delta x_{l}\right) \mathfrak{p}_{>} x_{>, i}\right]\right) \psi(\tilde{x}) .
\end{aligned}
$$

So, we found out the structure of constraints and wrote down the evolution operator in the same way as in section 2. It isn't surprising remembering results of sections 3 and 4 . Now we are ready to prove this statement without fixing any special coordinate system. We just need to find out the general formula for $\vec{v}_{\perp}$. Notice that by definition $\mathfrak{p}^{\mu}$ lies in a hyperplane of $x_{, 0}^{\mu}$ and $x_{, i}^{\mu}$. Then, due to the constraints (14), $\mathfrak{p}^{\mu}$ is proportional to $x_{\perp, 0}^{\mu}$. We have 
$v^{\mu}=\frac{\partial x^{\mu}\left(x^{0}, \sigma_{i}\right)}{\partial x^{0}}=\frac{x_{, 0}^{\mu}}{x_{, 0}^{0}}$ and hence $\mathfrak{p}^{\mu}$ is also proportional to $v_{\perp}^{\mu}: v_{\perp}^{\mu}=\alpha \mathfrak{p}^{\mu}$. To find the coefficient $\alpha$ we take $v_{\perp}^{\mu} \mathfrak{p}_{\mu}=\frac{x_{\perp, 0}^{\mu} \mathfrak{p}_{\mu}}{x_{, 0}^{0}}=\frac{-\gamma \sqrt{(-1)^{p} g}}{x_{, 0}^{0}}$ (the last equality is just the Euler's homogeneous function theorem) and $\mathfrak{p}^{\mu} \mathfrak{p}_{\mu}=(-1)^{p} \gamma^{2} \zeta(x)$. But $v_{\perp}^{\mu} \mathfrak{p}_{\mu}=\alpha \mathfrak{p}^{\mu} \mathfrak{p}_{\mu}$, hence we have

$$
v_{\perp}^{\mu}=-\frac{\sqrt{(-1)^{p} g}}{(-1)^{p} \gamma \zeta x_{, 0}^{0}} \mathfrak{p}^{\mu}
$$

and $1-\vec{v}_{\perp}^{2}=v_{\perp}^{\mu} v_{\mu \perp}=\frac{(-1)^{p} g \mathfrak{p}^{2}}{\gamma^{2} \zeta^{2} x_{, 0}^{0}}=\frac{g}{\zeta x_{, 0}^{0}}$, SO

$$
\int d x^{0} d V \sqrt{1-\vec{v}_{\perp}^{2}}=\int x_{, 0}^{0} d \sigma_{0} \sqrt{\left|\operatorname{det} x_{, i}^{\mu} x_{\mu, k}\right|} d^{p} \sigma_{i} \sqrt{\frac{g}{\zeta x_{, 0}^{0^{2}}}}=\int d^{p+1} \sigma \sqrt{|g|} .
$$

We proved (8) and the main statement of sections 3 and 4 for the most general case. 


\section{References.}

1. Krausz F. Path integral for a scalar propagator: Preprint HUTP 80-A003. Cambridge, 1980.

2. Fiziev P.P. Theor.Math.Phys., 62, p. 123 (1985).

3. Prokhorov L.V., Nuramatov A.G. Vestnik Leningr. Univ. (Ser. 4) 3 (18), p. 86 (1991). (in Russian)

4. Prokhorov L.V., Shabanov S.V. Gamiltonova mekhanika kalibrovochnikh sistem. Izd. SPbGU, 1997. (in Russian)

5. Dirac P.A.M. Lectures on quantum mechanics. Dover, 2001.

6. Barbashov B.M., Nesterenko V.V. Introduction to the relativistic string theory. World Scientific, 1990.

7. Golovnev A.V., Prokhorov L.V. Vestnik SPb Univ. (Ser.4), 2 (12), p. 86 (2003). (in Russian)

8. Green M.B., Shwartz J.H., Witten E. Superstring theory, vols. 1 and 2, Cambridge University Press, 1986.

9. Kaku M. Introduction to superstrings. Springer-Verlag, 1988.

10. Goto T. Prog.Theor.Phys, 46, p. 1560 (1971).

11. Hara O. Prog.Theor.Phys, 46, p. 1549 (1971). 